\title{
Optimization of perfluorocarbon emulsion properties for enhancing oxygen mass transfer in a bio-artificial liver support system
}

\author{
F.S. Moolman ${ }^{\mathrm{a}, *}$, H. Rolfes ${ }^{\mathrm{b}}$, S.W. van der Merwe ${ }^{\mathrm{c}}$, W.W. Focke ${ }^{\mathrm{a}}$ \\ a Department of Chemical Engineering, University of Pretoria, Pretoria 0001, South Africa \\ ${ }^{\mathrm{b}}$ Center for Polymer Technology, CSIR, P.O. Box 395, Pretoria 0001, South Africa \\ ${ }^{\mathrm{c}}$ Department of Internal Medicine, University of Pretoria, Pretoria 0001, South Africa
}

Received 16 May 2003; received in revised form 7 January 2004; accepted 4 February 2004

\begin{abstract}
The oxygen carrying performance of a perfluorooctyl bromide (PFOB) emulsion is considered. The intended purpose is to enhance hepatocyte growth and function in a bio-artificial liver support system (BALSS). Such oxygen carrying emulsions have previously been used in biological systems (e.g. cell culturing). However, optimum emulsion characteristics for enhanced oxygen mass transfer have not been established nor was consideration given to the effect of emulsion rheology on mass transfer: With increase in the dispersed phase volume fraction $\left(\phi_{\mathrm{p}}\right)$ both the oxygen holding capacity and the viscosity increases. These issues are addressed here using simplified mass transfer models, amenable to analytical solution, for both gas-sparged and membrane oxygenators. The model predictions that the rate at which oxygen can be supplied improves with increase in $\phi_{\mathrm{p}}$ and decrease in emulsion droplet size were checked experimentally for perfluorooctyl bromide emulsions. Biological applications mandate a suitably low emulsion viscosity and this limits the usable range for the PFOB volume fraction to $\phi_{\mathrm{p}}<0.5$. There is also a lower practical limit to the possible droplet size (about $100 \mathrm{~nm}$ ).

The predicted higher oxygen loading rates for the membrane oxygenator compared to the gas-sparged oxygenator was also confirmed by experiment. Predicted and measured volumetric mass transfer coefficients $(k \times a)$ were ca. $8 \times 10^{-4} \mathrm{~s}^{-1}$ for the gas-sparged oxygenator and ca. $1 \mathrm{~s}^{-1}$ for the hollow fibre membrane unit at 20 vol.\% PFOB emulsions.

(C) 2004 Elsevier B.V. All rights reserved.
\end{abstract}

Keywords: Bio-artificial liver; Optimization; Mass transfer; Emulsion; Perfluorocarbon; Oxygen transfer

\section{Introduction}

Acute fulminant liver failure is a potentially life threatening condition with mortality rates exceeding $80 \%$ [1]. Orthotopic liver transplantation is currently the only effective treatment. The shortage of suitable donors and the short time span between onset of acute liver failure and death contributes to the high mortality seen in this condition. Mortality rate could be reduced if a therapy were available capable of supporting liver functions, either until patient recovery or as a bridge to liver transplantation. The development of such a therapy can take the form of an artificial, biological or bio-artificial approach. Due to the complexity of the liver, bio-artificial approaches are the most promising. Bio-artificial liver support systems (BALSS) must comply with a number of requirements including immune protection of the hepatocytes (liver cells) in the bioreactor and efficient

\footnotetext{
* Corresponding author. Tel.: +27-12-841-4212; fax: +27-12-841-3553. E-mail address: smoolman@csir.co.za (F.S. Moolman).
}

mass transfer between the cells and the contact fluid from the patient.

The BALS system under development at the University of Pretoria (UP) and the CSIR is shown schematically in Fig. 1 [2]. It allows for direct hepatocyte-plasma contact and this necessitates removal of the immunologically active white blood cells. This approach is also used in the spiral-wound polyester matrix system of the University of Amsterdam $[3,4]$. Thus, in the first step, the blood plasma is separated from the cellular blood components. It is unfortunate that red blood cells are also removed in the process: owing to the absence of red blood cells and the low solubility of $\mathrm{O}_{2}$ in plasma, the oxygen supply to the hepatocytes in the bioreactor is insufficient. The UP-CSIR BALSS addresses this problem by the introduction of an oxygen carrier into the plasma circulation system. The oxygen carrier-plasma blend is oxygenated using a neonatal membrane unit, before flowing through the bioreactor that contains a three-dimensional scaffold with attached hepatocytes. The oxygen carrier (or 'blood substitute') is separated from the treated plasma before it is returned to the patient. 


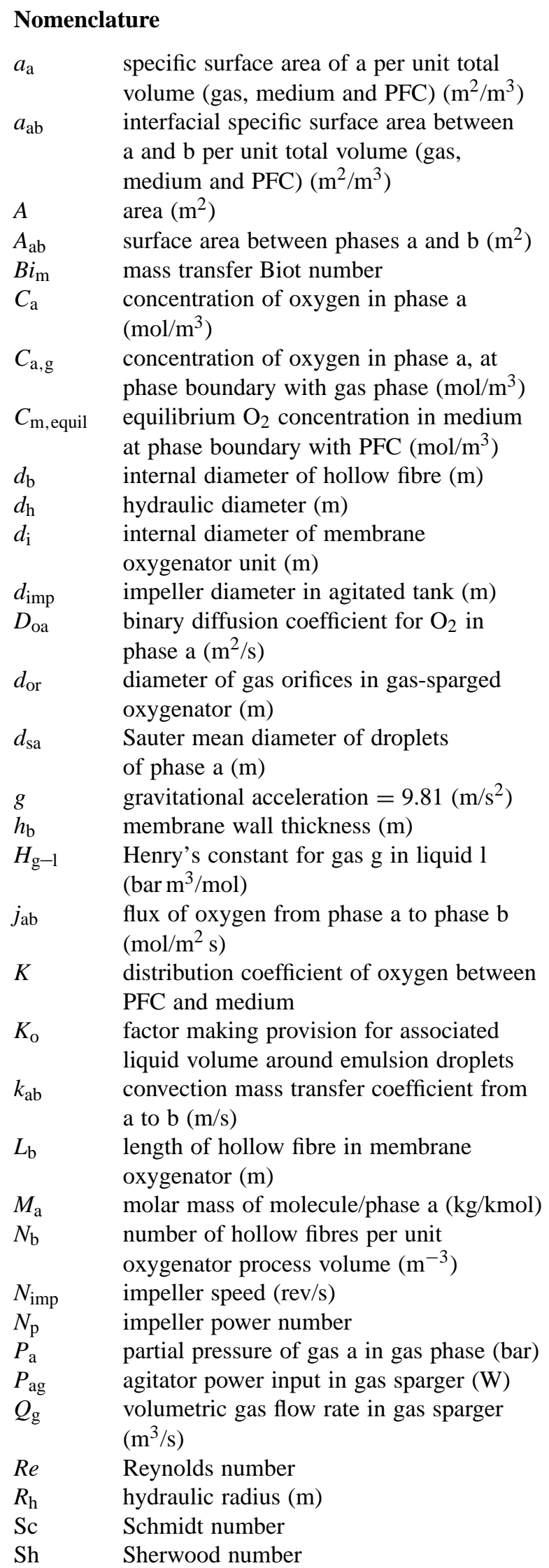

$a_{\mathrm{a}} \quad$ specific surface area of a per unit total volume (gas, medium and PFC) $\left(\mathrm{m}^{2} / \mathrm{m}^{3}\right)$

\begin{tabular}{|c|c|}
\hline$T$ & temperature $(\mathrm{K})$ \\
\hline$t_{\mathrm{m}}$ & $\begin{array}{l}\text { average time spent in membrane oxygenator } \\
\text { by unit cell of emulsion (s) }\end{array}$ \\
\hline$u_{\mathrm{e}}$ & bulk (average) emulsion velocity $(\mathrm{m} / \mathrm{s})$ \\
\hline$u_{\mathrm{or}}$ & $\begin{array}{l}\text { gas bubble velocity through orifice } \\
\text { (gas-sparged oxygenator) }(\mathrm{m} / \mathrm{s})\end{array}$ \\
\hline$V_{\mathrm{gs}}$ & $\begin{array}{l}\text { total system volume in gas-sparged } \\
\text { oxygenator }=V_{\mathrm{p}}+V_{\mathrm{g}}+V_{\mathrm{m}}\left(\mathrm{m}^{3}\right)\end{array}$ \\
\hline$V_{\mathrm{ob}}$ & $\begin{array}{l}\text { molar volume of liquid oxygen at its } \\
\text { boiling point }\left(\mathrm{m}^{3} / \mathrm{mol}\right)\end{array}$ \\
\hline$V_{\mathrm{t}}$ & $\begin{array}{l}\text { total system volume in membrane } \\
\text { oxygenator }=V_{\mathrm{p}}+V_{\mathrm{gb}}+V_{\mathrm{m}}+V_{\mathrm{b}}\left(\mathrm{m}^{3}\right)\end{array}$ \\
\hline \multicolumn{2}{|c|}{ Greek symbols } \\
\hline$\delta_{\mathrm{c}}$ & "Film" theory boundary layer thickness (m) \\
\hline$\phi_{\mathrm{g}}$ & $\begin{array}{l}\text { volume fraction gas in } \\
\text { medium }=V_{\mathrm{g}} /\left(V_{\mathrm{p}}+V_{\mathrm{g}}+V_{\mathrm{m}}\right)\end{array}$ \\
\hline$\phi_{\mathrm{p}}$ & $\begin{array}{l}\text { volume fraction PFOB in medium, exclusive } \\
\text { of gas }=V_{\mathrm{p}} /\left(V_{\mathrm{p}}+V_{\mathrm{m}}\right)\end{array}$ \\
\hline$\eta$ & dynamic viscosity (Pas) \\
\hline$\eta_{\mathrm{r}}$ & reduced viscosity $=\eta_{\mathrm{e}} / \eta_{\mathrm{m}}(\mathrm{Pas})$ \\
\hline$\rho$ & density $\left(\mathrm{kg} / \mathrm{m}^{3}\right)$ \\
\hline$\sigma_{\mathrm{s}}$ & surface tension $(\mathrm{N} / \mathrm{m})$ \\
\hline$\tau_{\mathrm{e}}$ & $\begin{array}{l}\text { emulsion residence time in membrane } \\
\text { oxygenator (s) }\end{array}$ \\
\hline \multicolumn{2}{|c|}{ General subscripts } \\
\hline ave & average \\
\hline $\mathrm{b}$ & membrane \\
\hline e & $\begin{array}{l}\text { emulsion phase (combined PFOB and } \\
\text { medium) }\end{array}$ \\
\hline equil & value of parameter at equilibrium \\
\hline $\mathrm{g}$ & gas phase \\
\hline $\mathrm{m}$ & medium (or plasma) phase \\
\hline $\mathrm{p}$ & PFOB phase \\
\hline $\mathrm{w}$ & property evaluated at the wall \\
\hline
\end{tabular}

'Artificial blood' or 'blood substitutes' have been under development for several decades [5]. These terms are misleading, as they actually refer to compounds that only perform the oxygen carrying function of blood; 'oxygen carrier' is more specific. Perfluorocarbons (PFCs) are a family of inert compounds with exceptionally high capacity to dissolve gasses [6]. Various studies [7-9] have demonstrated their ability to improve cell growth and yield in cell culture systems. As PFCs are immiscible with aqueous media, they need to be emulsified for use as oxygen carriers in such media. Kraft et al. [14] give a review of the status of PFC emulsification technology. Lipids, e.g. as egg yolk lecithin (L- $\alpha$-phosphatidylcholine) are suitable emulsifiers [10]. PFC emulsions with Sauter mean droplet diameters (SMD) of ca. $0.2 \mu \mathrm{m}$ are best suited for oxygenation in biological applications [11-13]. Perfluorooctyl bromide (PFOB) is the most commonly used PFC owing to its low toxicity and 


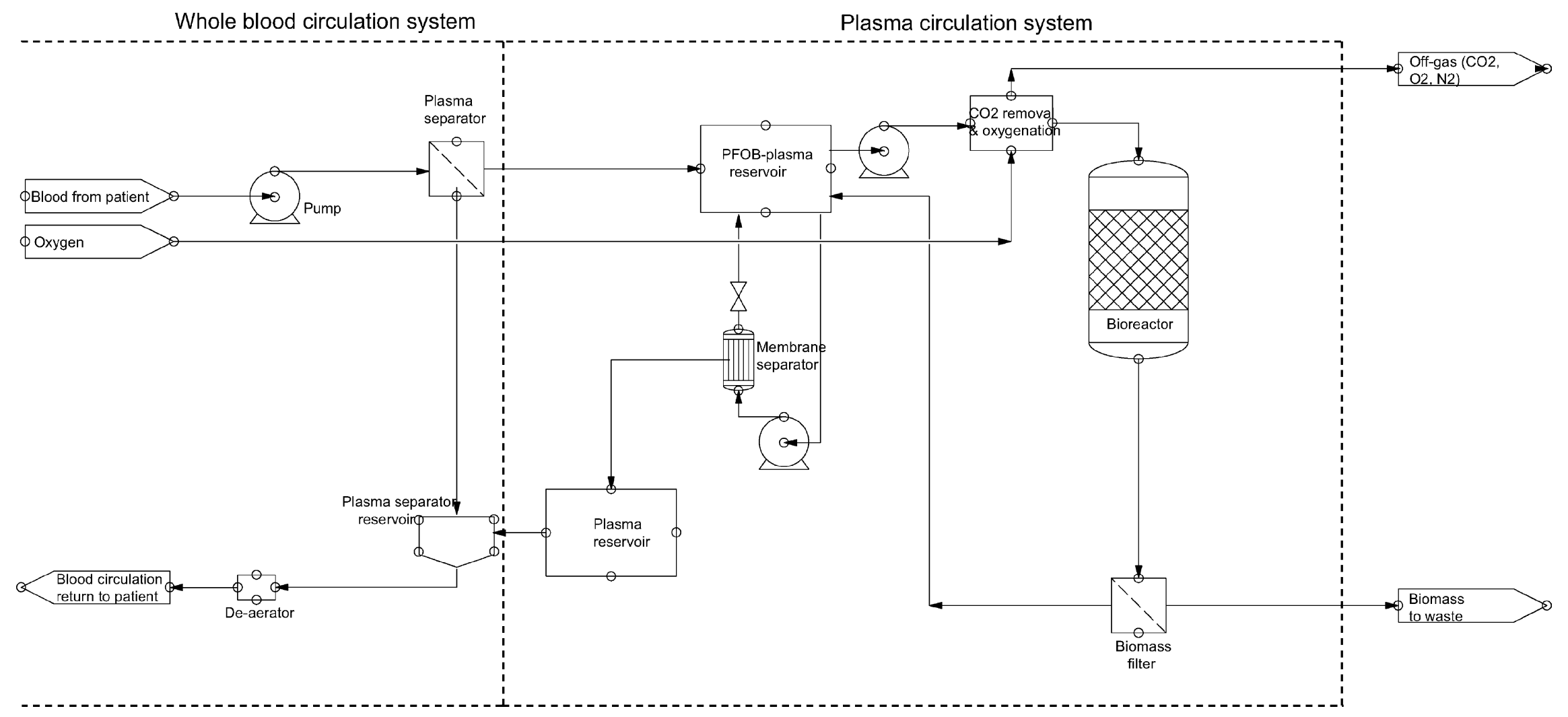

Fig. 1. Basic flow diagram of the UP-CSIR BALSS showing the whole-blood and plasma circulation systems. 
its rapid elimination from the reticulo-endothelial system. The slightly lipophilic character also provides for improved emulsion stability.

The mass transfer of gasses to and from the emulsion droplets is of vital importance in PFC oxygen carrier applications. The mass transfer characteristics are key in tissue oxygenation and cell culture systems, specifically in the design and modelling of BALS systems. Important aspects include $\mathrm{O}_{2}$ loading times at typical circulation rates and emulsion viscosity. This study investigates two PFOB emulsions of different Sauter mean droplet diameters $(0.2$ and $4 \mathrm{~mm})$ and examines the influence of parameters such as droplet size, dispersed phase volume fraction, emulsion viscosity, etc. on oxygen carrier properties.

\section{Model development}

Mass transfer models are developed for two different oxygenator types: membrane and gas-sparged. Comprehensive details are reported in [15]. Appendices A and B summarize the determination of auxiliary coefficients for the models.

Losses of PFOB into the gas phase were neglected considering the low $\mathrm{PFOB}$ vapour pressure $\left(590 \mathrm{~Pa}\right.$ at $20^{\circ} \mathrm{C}$ and $1400 \mathrm{~Pa}$ at $37^{\circ} \mathrm{C} \mathrm{[19])} \mathrm{and} \mathrm{the} \mathrm{fact} \mathrm{that} \mathrm{it} \mathrm{is} \mathrm{emulsified.} \mathrm{It}$ was also assumed that the oxygen partial pressure in gas bubbles remained constant and that PFOB droplet density is independent of the dissolved oxygen concentration.

\subsection{Mass transfer modelling}

Modelling gas absorption into emulsions in oxygenators is complex owing to the multiphase nature of the system. It is well established that the gas-liquid mass transfer rate is significantly enhanced when the solubility of the gas is substantially higher in the dispersed phase and when the dispersed droplets are small compared to the (film theory) mass transfer boundary layer thickness $d_{c}$ [38]. Such enhancement of the adsorption rate has been explained in terms of the so-called "microphase catalysis"-, "grazing"or "shuttle"- mechanism [17,39]. In terms of this hypothesis the emulsion droplets exchange rapidly between the bulk region and the stagnant mass transfer boundary layer zone located at the gas-liquid interface. The droplets have a substantially higher capacity for gas solubilization. This, combined with the preferential dissolution of the gas in the droplets, reduces the local gas concentration in the medium. In effect the concentration gradient in the liquid medium near the interface is increased and gas absorption is enhanced. Furthermore, direct contact between the gas and dispersed phases might provide another mechanism contributing to mass transfer enhancement [37].

Brilman et al. [37,38] and Beenackers and van Swaaij [39] reviewed the plethora of mass transfer models proposed for such gas-liquid-liquid systems. The model chosen in this study is of the homogeneous type. Despite the fact that such models are based on physically unrealistic assumptions, they do predict the correct trends with respect to changing operating conditions such as the gas-liquid contact time, the relative solubility and the dispersed phase volume fraction [38]. An added advantage is the mathematical simplicity that permits analytical solution of the differential equations. The following simplifying assumptions, common to most homogeneous models, are made [38]:

- "Film" theory describes mass transfer to the medium. "Film" theory assumes (a) that the gas diffuses from the interface into the liquid medium through a hypothetical stagnant film; (b) a linear concentration gradient in the boundary layer; and (c) a sharp transition at the edge of the stagnant film to a well-mixed bulk fluid where the oxygen concentration gradient is negligible owing to bulk fluid mixing and the Brownian motion of emulsion droplets.

- The emulsion droplets are small with respect to the "film"-theory boundary layer thickness. Typical concentration boundary layers in oil-in-water emulsions have thicknesses exceeding 5-10 $\mu \mathrm{m}[8,38]$ whereas the emulsions studied presently have droplet diameters of either $0.2 \mu \mathrm{m}$ or $4 \mu \mathrm{m}$.

- The emulsified phase is treated as a continuum and is homogeneously dispersed throughout the continuous phase. This allows the use of lumped-parameter mass transfer coefficients.

- Mass transfer resistances within the emulsion droplets are negligible. According to Ju et al. [16], the presence of surfactant molecules at the PFC droplet surfaces does not affect oxygen transport into the PFOB-plasma phase. This assumption is further justified by the small size of the droplets [17] and the fact that the estimated mass transfer Biot number is significantly less than unity [15]. Consequently the gas concentration within the PFOB droplets can be taken as being uniform.

- Rapid exchange between droplets within the boundary layer and the bulk [37]. This implies that the gas concentrations inside the PFOB droplets in the bulk emulsion and inside the boundary layer phase are the same. Thus only one PFOB concentration is relevant in the mass transfer model.Mass transfer enhancement can be modelled by an apparent direct transfer of gas to the first layer of emulsion droplets at the gas-liquid interface. Conventional homogeneous models ignore the possibility of direct droplet (membrane) gas contact and assume that mass transport occurs only through the continuous phase, i.e.:

gas $\leftrightarrow$ membrane $\leftrightarrow$ medium $\leftrightarrow$ PFOB droplet

In the present model the mass transfer enhancement is accounted for empirically by postulating that direct oxygen transfer to PFOB droplets can take place inside the boundary layer:

gas $\leftrightarrow$ membrane $\leftrightarrow$ PFOB 


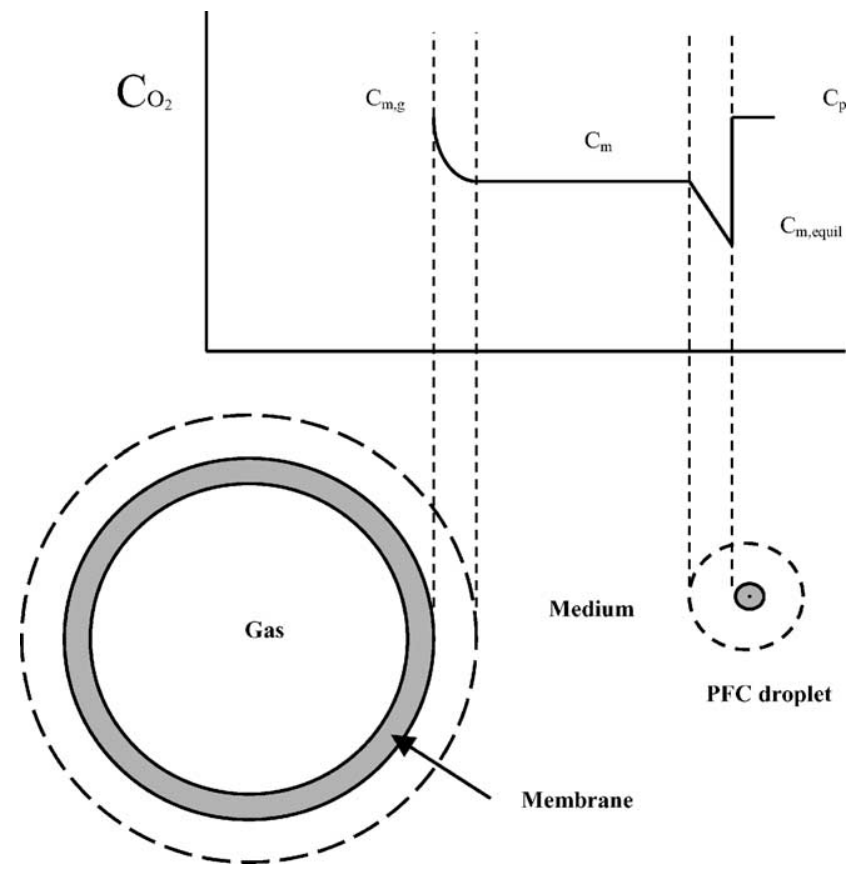

Fig. 2. Schematic representation of oxygen transfer from gas to PFC for a single hollow fibre in a hollow fibre membrane oxygenator.

Droplets located closest to the gas liquid interface have by far the greatest effect on mass transfer enhancement [37]. For this reason it is expedient to assume that the enhancement is limited to a single layer of emulsion droplets at the interface.

\subsection{Membrane oxygenator model}

The membrane oxygenator consists of a bundle of hollow fibres connected at their ends by manifolds. The inside of the fibres is filled with pressurized gas while the space outside the fibre bundle is perfused with PFOB emulsion. The hydrophobic nature of the membranes implies that voids within the membrane are filled with gas [20]. Furthermore, it is assumed that the permeability of the membrane material is very high. Accordingly the mass transfer resistance on the fluid side is assumed rate controlling.

The model further assumes plug flow through the oxygenator and neglects axial diffusion effects. Fig. 2 shows a schematic representation of the oxygen transfer from gas to PFOB for a single hollow fibre. The discontinuity in the concentration at the phase boundary between the medium and the PFOB is due to the higher solubility of oxygen in the latter. An oxygen mole balance over a unit cell of medium, using the concentrations as defined in Fig. 2, yields: [accumulation of oxygen in medium]

$=[$ flow into medium from membrane $]$

- [flow out of medium into PFOB]

$V_{\mathrm{m}} \frac{\mathrm{d} C_{\mathrm{m}}}{\mathrm{d} t_{\mathrm{m}}}=A_{\mathrm{bm}} j_{\mathrm{bm}}-A_{\mathrm{mp}} j_{\mathrm{mp}}$

The surface areas and fluxes in Eq. (1) can be calculated as follows:

$$
\begin{aligned}
& A_{\mathrm{mp}}=V_{\mathrm{t}} a_{\mathrm{mp}} \quad A_{\mathrm{bm}}=V_{\mathrm{t}} a_{\mathrm{bm}} \quad j_{\mathrm{mp}}=k_{\mathrm{mp}}\left(C_{\mathrm{m}}-C_{\mathrm{m}, \text { equil }}\right) \\
& j_{\mathrm{bm}}=k_{\mathrm{bm}}\left(C_{\mathrm{m}, \mathrm{g}}-C_{\mathrm{m}}\right)
\end{aligned}
$$

Substituting Eq. (2) into Eq. (1):

$$
\begin{aligned}
\frac{\mathrm{d} C_{\mathrm{m}}}{\mathrm{d} t_{\mathrm{m}}}= & \frac{V_{\mathrm{t}}}{V_{\mathrm{m}}}\left[k_{\mathrm{bm}} a_{\mathrm{bm}}\left(C_{\mathrm{m}, \mathrm{g}}-C_{\mathrm{m}}\right)\right. \\
& \left.-k_{\mathrm{mp}} a_{\mathrm{mp}}\left(C_{\mathrm{m}}-C_{\mathrm{m}, \text { equil }}\right)\right]
\end{aligned}
$$

An analogous equation is obtained for the dispersed phase by performing a similar oxygen mole balance:

$$
\begin{aligned}
\frac{\mathrm{d} C_{\mathrm{p}}}{\mathrm{d} t_{\mathrm{m}}}= & \frac{V_{\mathrm{t}}}{V_{\mathrm{p}}}\left[k_{\mathrm{mp}} a_{\mathrm{mp}}\left(C_{\mathrm{m}}-C_{\mathrm{m}, \text { equil }}\right)\right. \\
& \left.+k_{\mathrm{bp}} a_{\mathrm{bp}}\left(C_{\mathrm{m}, \mathrm{g}}-C_{\mathrm{m}, \text { equil }}\right)\right]
\end{aligned}
$$

Eqs. (3) and (4) describe the time-dependent mass transfer to a fluid element as it traverses the membrane oxygenator. They form a coupled set of linear first order ordinary differential equations [21] that can be solved analytically [15]. Except for $k_{\mathrm{bp}}$ that is treated as an adjustable empirical constant, all parameters can either be estimated or measured directly (see Appendices A and B).

A maximum Reynolds number of ca. 140 was estimated for the lowest viscosity emulsion at maximum allowable flow rate through the oxygenator. Thus laminar flow conditions will prevail for all allowable flow rates.

Three different physical property calculation procedures were explored for the membrane oxygenator model to determine their effect on system performance predictions [15]: Newtonian flow correlations with properties evaluated at either (a) average conditions or (b) the high shear conditions at the wall as recommended by Cussler [23]; and (c) non-Newtonian power law equations. For the latter the Metzner-Reed Reynolds number for power law fluids was used in the calculations [22]. Appendix B details the correlations that were used.

\subsection{Gas-sparged oxygenator model}

Proceeding in a similar way, an analogous set of simultaneous differential equations is obtained for the gas-sparged oxygenator:

$$
\begin{aligned}
\frac{\mathrm{d} C_{\mathrm{m}}}{\mathrm{d} t} & =\frac{1}{1-\phi_{\mathrm{p}}\left(1-\phi_{\mathrm{g}}\right)-\phi_{\mathrm{g}}}\left[k_{\mathrm{gm}} a_{\mathrm{gm}}\left(C_{\mathrm{m}, \mathrm{g}}-C_{\mathrm{m}}\right)-k_{\mathrm{mp}} a_{\mathrm{mp}}\left(C_{\mathrm{m}}-C_{\mathrm{m}, \text { equil }}\right)\right] \\
\frac{\mathrm{d} C_{\mathrm{p}}}{\mathrm{d} t} & =\frac{1}{\phi_{\mathrm{p}}\left(1-\phi_{\mathrm{g}}\right)}\left[k_{\mathrm{mp}} a_{\mathrm{mp}}\left(C_{\mathrm{m}}-C_{\mathrm{m}, \text { equil }}\right)+k_{\mathrm{gp}} a_{\mathrm{gp}}\left(C_{\mathrm{p}, \mathrm{g}}-C_{\mathrm{p}}\right)\right]
\end{aligned}
$$


Appendix A lists procedures for estimating or measuring the model parameters. The mass transfer coefficient, $k_{\mathrm{gp}}$, is treated as an adjustable empirical constant.

\subsection{Emulsion viscosity correlation}

The effect of $\phi_{\mathrm{p}}$ on the viscosity of dilute emulsions is described by the well-known Einstein equation [24]:

$\eta_{\mathrm{r}}=1+2.5 \phi_{\mathrm{p}}$

Mandersloot and Scott [24] proposed an improvement to the Einstein viscosity equation that is valid for both dilute and concentrated emulsions:

$\eta_{\mathrm{r}}=\left[1-\frac{\phi_{\mathrm{p}}}{\phi_{\mathrm{p}, \max }}\right]^{-2.5 k_{\mathrm{o}} \phi_{\mathrm{p}, \max }}$

Here $\phi_{\mathrm{p}, \max }$ is the maximum attainable dispersed phase volume fraction for the specific emulsion system. The factor $k_{\mathrm{o}}$ accounts for the presence of liquid associated with droplets or droplet aggregates that increases the effective volume of the dispersed phase [24].

\section{Materials and methods}

Emulsions were prepared using 98\% PFOB (ABCR $\mathrm{GmbH}$ ), demineralised water, egg yolk lecithin (Lipoid E80S-Lipoid AG), sodium chloride (Merck), sodium bicarbonate (BDH Chemicals) and Vitamin E (BASF) as anti-oxidant. All ingredients, except PFOB, were added to water and homogenized for $5 \mathrm{~min}$ at $6000 \mathrm{rpm}$ using a rotor-stator-type homogenizer (Silverson L4R). The PFOB was then added drop wise over $15 \mathrm{~min}$ while continuing the stirring at $6000 \mathrm{rpm}$. Final emulsification was then achieved by either ultrasonication or high-pressure homogenization. The $4 \mu \mathrm{m}$ Sauter mean droplet diameter emulsion was prepared by ultrasonication in an Ultrasonic Homogenizer 4710 series (Cole-Parmer Instrument Co.) set at maximum power $(120 \mathrm{~W})$. The ingredients were sonicated for $10 \mathrm{~min}$, homogenized for $7 \mathrm{~min}$, sonicated for $3 \mathrm{~min}$ followed by ten repetitions composed of 2 min sonications together with 1 min breathers.

The $0.2 \mu \mathrm{m}$ Sauter mean droplet diameter emulsion was obtained by high-pressure homogenization in an APV 2000 Laboratory Homogenizer using 15 passes at 1800 bar. An ice bath was used to cool the emulsion during the homogenization in an effort to prevent degradation of the ingredients. Nitrogen was continuously bubbled through the emulsion to prevent oxidative degradation of the lecithin surfactant [25].

The homogenized emulsions were autoclaved at $121^{\circ} \mathrm{C}$ for $15 \mathrm{~min}$ and stored refrigerated in sealed containers. Droplet size distributions were determined, before and after autoclaving, using a Malvern 2000 Mastersizer laser scattering particle size distribution analyzer. Emulsion stability was determined by following changes in droplet size
Table 1

Selected specifications of Polystan Safemicro Neonatal Oxygenator

\begin{tabular}{lc}
\hline Parameter & Value \\
\hline Total hollow fibre surface area $\left(\mathrm{m}^{2}\right)$ & 0.33 \\
Fibre inner diameter $(\mu \mathrm{m})$ & 280 \\
Fibre wall thickness $(\mu \mathrm{m})$ & 50 \\
Fibre length $(\mathrm{mm})$ & 73 \\
Process volume $(\mathrm{ml})$ & 26 \\
Maximum process flow rate $\left(\mathrm{ml} \mathrm{s}^{-1}\right)$ & 13.3 \\
\hline
\end{tabular}

distributions over time: these were measured directly after manufacture and after 1 month of storage at room temperature. Measuring coalescence kinetics of the emulsions with a Malvern Turbiscan MA 2000 machine also provided a stability check.

Viscosity was measured using a Rheometric Scientific SR5 parallel plate rheometer with a $25 \mathrm{~mm}$ plate diameter and a $0.5 \mathrm{~mm}$ gap. A parallel plate configuration was used as it is recommended for characterizing emulsion rheology [26]. Measurements were done at $37^{\circ} \mathrm{C}$ using step stress tests carried out at shear rates ranging from 15 to $1000 \mathrm{~s}^{-1}$. Shear rate was kept constant for $200 \mathrm{~s}$ during each step.

Fig. 3(a) shows the gas-sparged oxygenator experimental setup. The PFOB emulsion was placed in a $500 \mathrm{ml}$ vessel fitted with a stirrer, sparger, copper heat exchange tubes (for temperature regulation) and an oxygen probe (Biological Oxygen Monitor, YSI Model 5300, YSI Incorporated, USA). The oxygenator was kept at $37^{\circ} \mathrm{C}$ using a water bath, circulation pump and heat exchange tubes. The flow rate was measured and the gas brought to temperature in the copper heat exchanger tubes placed inside a water bath before it was sparged through the emulsion. The exiting gas was passed through a flow meter and an oxygen analyzer. Before each experimental run the emulsion was de-oxygenated with nitrogen. The oxygen loading profile vs. time was determined by bubbling oxygen through the emulsion at a fixed rate.

Fig. 3(b) shows the membrane oxygenator experimental setup. The emulsion was continuously de-oxygenated in a reservoir container by bubbling nitrogen through it. It was then pumped through a heat exchanger before passing through the membrane oxygenator. A Polystan Safemicro Neonatal Oxygenator combination heat exchangermembrane oxygenator was used (Table 1). The oxygenloaded emulsion was collected in a container where the oxygen content was measured with an oxygen probe (Biological Oxygen Monitor, YSI Model 5300, YSI Incorporated, USA). The emulsion was pumped through the oxygenator at a fixed rate, and the outlet oxygen concentration measured until it stabilized. This stabilized reading was taken as the oxygen concentration in the emulsion for the specific residence time determined by the pumping rate. Each experiment yielded a single data point. The full oxygen loading curve for a given emulsion was generated by varying the pumping speed. Table 2 lists typical parameter values and settings for the gas-sparged and membrane oxygenators units during the experiments. 


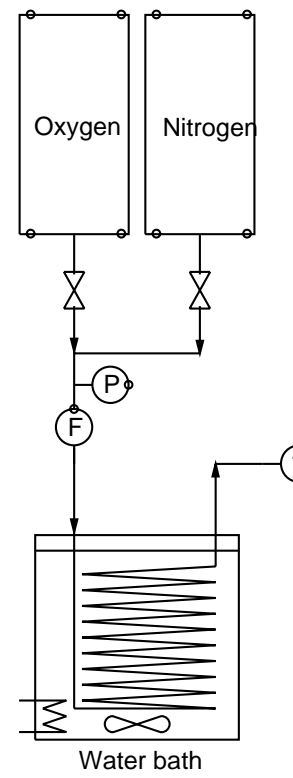

(a)

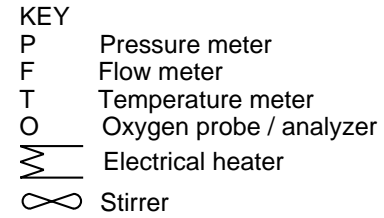

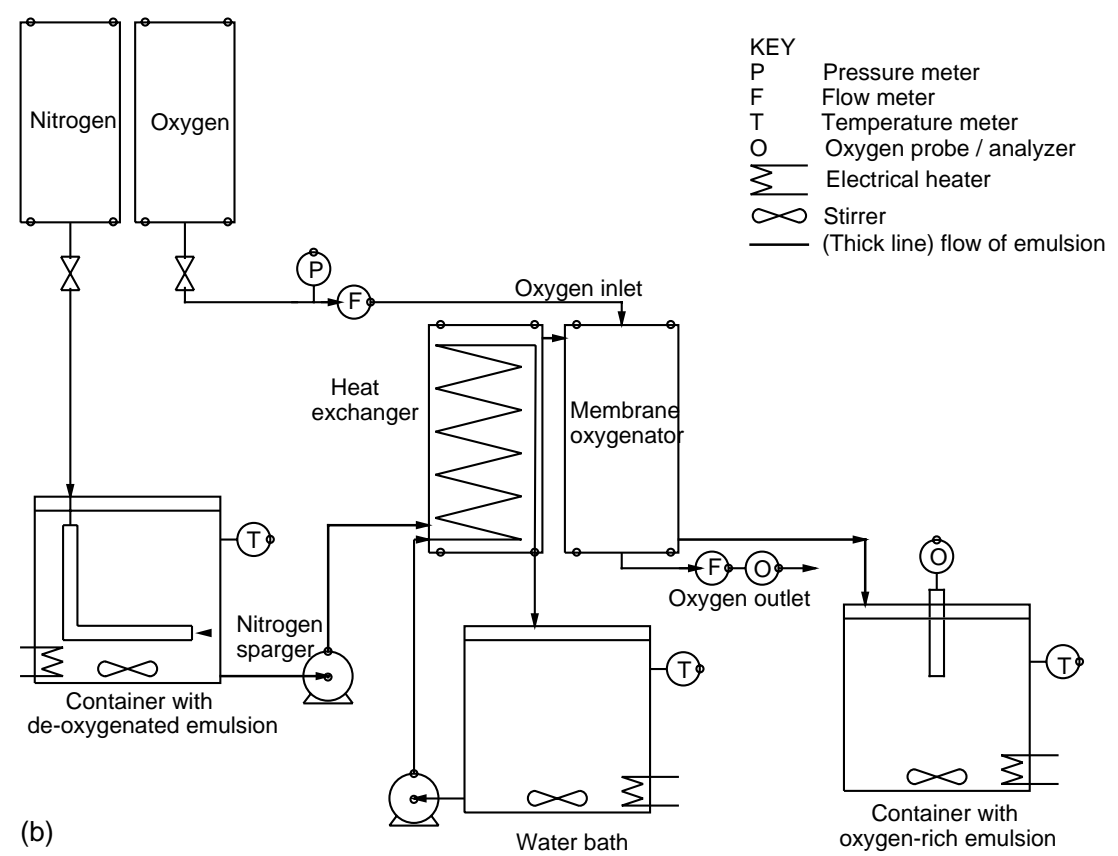

Fig. 3. (a). Gas-sparged oxygenator experimental setup. (b). Membrane oxygenator experimental setup.

Mass transfer coefficients were estimated using correlations from literature (see Appendices A and B). The oxygenator models were fitted by regression of the experimental data obtained for the $0.2 \mu \mathrm{m}$ droplet size emulsion at $20 \%$ volume fraction only. The only adjustable parameters were the mass transfer coefficients for gas transport from interface to droplet in the boundary layer $\left(k_{\mathrm{bp}}\right.$ and $\left.k_{\mathrm{gp}}\right)$. Model predictions were then made for other droplet sizes, dispersed phase volume fractions and gas flow rates keeping this parameter unchanged.

\section{Results}

Fig. 4 shows the droplet size distributions for the 0.2 and $4 \mu \mathrm{m}$ emulsions. The high-pressure homogenized emulsion remained stable for several months. The sonicated emulsions showed a tendency to destabilize after about 1 week of storage.

Fig. 5 shows that the Mandersloot viscosity correlation, Eq. (7), provides an excellent fit for three different viscosity data sets with $k_{\mathrm{o}}=1.38$ and $\phi_{\mathrm{p}, \max }=0.60$. The 
Table 2

Typical parameters for gas-sparged and membrane oxygenators used in the models and experiments

\begin{tabular}{lc}
\hline Parameter & Value \\
\hline Gas-sparged oxygenator & 0.85 \\
Oxygen pressure (bar) & 0.48 \\
Volume of oxygenator $(1)$ & 0.075 \\
Oxygenator diameter $(\mathrm{m})$ & 0.5 \\
Gas flow rate $(1 / \mathrm{min})$ & 5 \\
Orifices & 1 \\
Orifice diameter $(\mathrm{mm})$ & 50 \\
Impeller diameter $(\mathrm{mm})$ & 2000 \\
Impeller rotational speed $(\mathrm{rpm})$ & \\
Membrane oxygenator & 0.85 \\
Oxygen pressure $(\mathrm{bar})$ & $4.15 \times 10^{7}$ \\
Hollow fibres/volume $\left(\mathrm{m}^{-3}\right)$ & 0.073 \\
Length of fibres $(\mathrm{m})$ & 280 \\
Fibre internal diameter $(\mu \mathrm{m})$ & 50 \\
Fibre wall thickness $(\mu \mathrm{m})$ & 0.5 \\
Fibre porosity & 0.33 \\
Total hollow fibre surface area $\left(\mathrm{m}^{2}\right)$ & 26 \\
Process volume $(\mathrm{ml})$ & 0.015 \\
Process fluid velocity $\left(\mathrm{m} \mathrm{s}^{-1}\right)$ & \\
\hline
\end{tabular}

value for the maximum packing volume fraction is within the 0.60-0.66 range observed for sheared flow of dispersions [24]. Note that the constant $k_{\mathrm{O}}$ was determined independently by fitting $\eta_{\mathrm{r}}=1+2.5 k_{\mathrm{o}} \phi_{\mathrm{p}}$ to experimental data for dilute emulsions [15].

The membrane oxygenator model predictions were not sensitive to the choice of the physical property calculation method. The results, using the three different approaches, agreed to within $2 \%$.

Data for the 20 vol.\% emulsions are shown in Figs. 6-9. Fig. 6 shows the correlation between oxygenator model predictions and experimental data for the $0.2 \mu \mathrm{m}$ emulsions. It is clear that the membrane oxygenator provides for significantly faster oxygen loading rates. The main reason for this

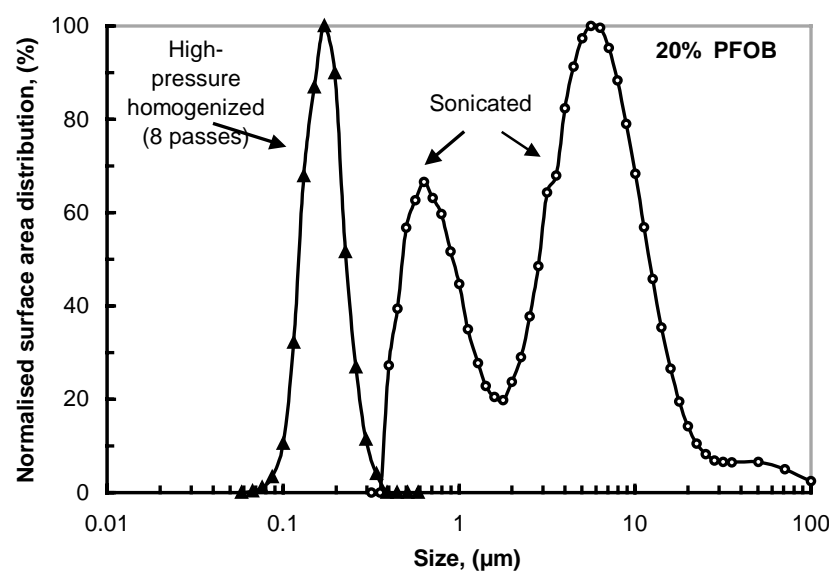

Fig. 4. The high-pressure homogenized emulsion has smaller droplets and a narrower droplet size distribution than the sonicated emulsion. The latter also exhibits a bimodal droplet size distribution.

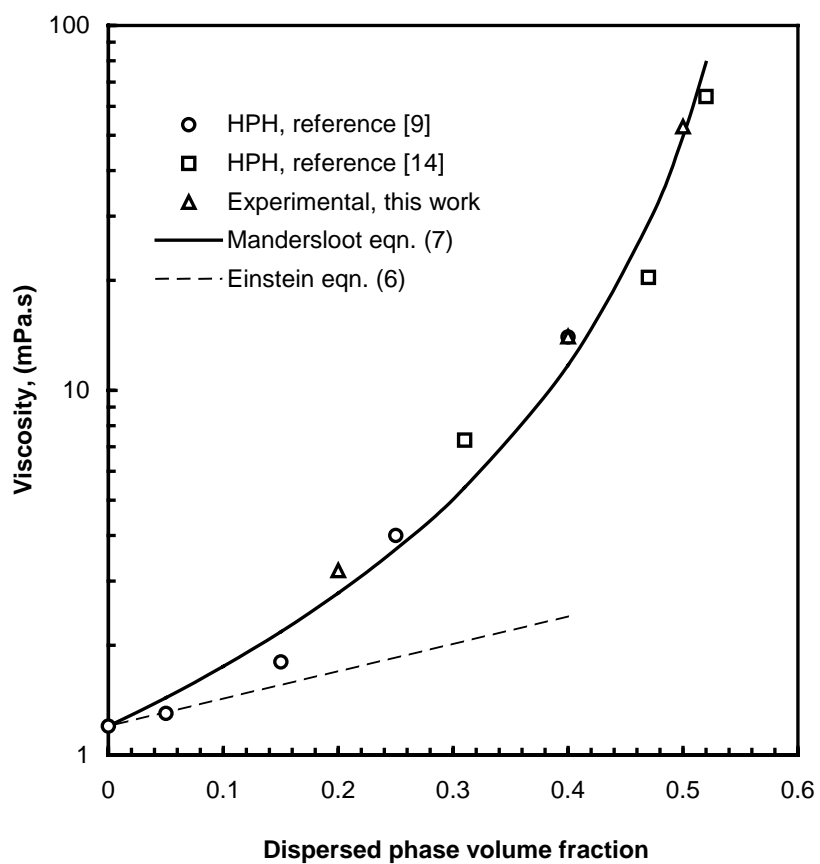

Fig. 5. The effect of dispersed phase volume fraction on emulsion viscosity.

is the much lower specific mass transfer surface area available in the gas-sparged system as compared to the membrane oxygenator: Whereas measured mass transfer coefficients were similar for the two oxygenators $\left(k \approx 1 \times 10^{-3} \mathrm{~m} / \mathrm{s}\right)$, the corresponding overall volumetric mass transfer coefficients were ca. $k \times a \approx 8 \times 10^{-4} \mathrm{~s}^{-1}$ and $k \times a \approx 1 \mathrm{~s}^{-1}$ for the gas-sparged and membrane oxygenators, respectively.

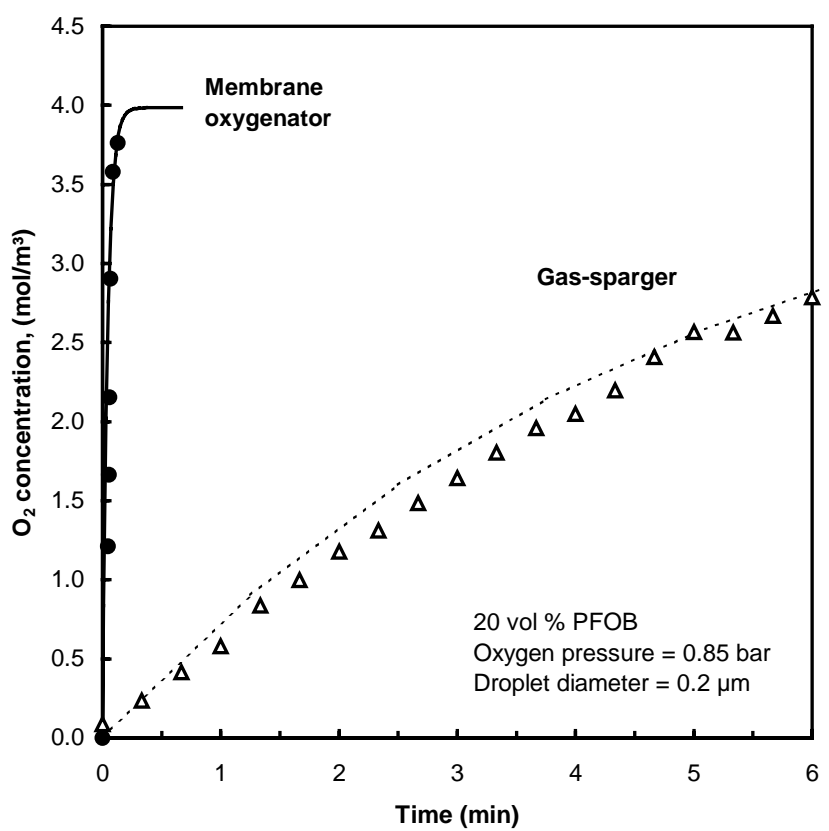

Fig. 6. Comparing the oxygen transfer rates in the gas-sparger and the membrane oxygenator. 


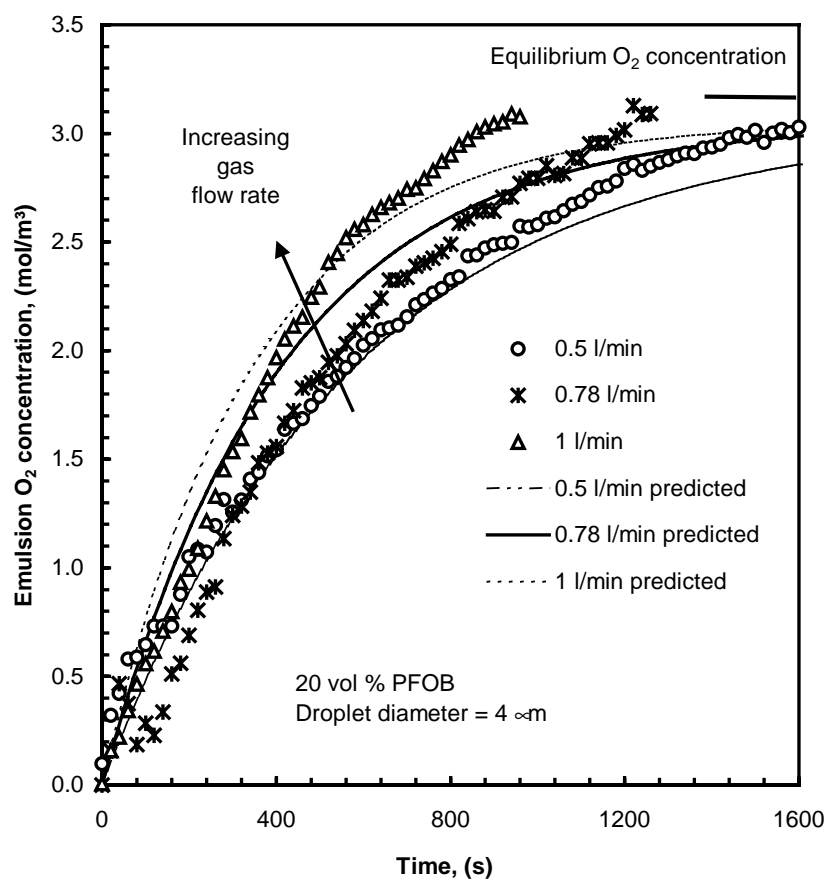

Fig. 7. Gas-sparged oxygenator: influence of gas flow rate on oxygen loading rate.

Fig. 7 shows that the $\mathrm{O}_{2}$ loading rate in the gas sparger increases with gas flow rate. Both oxygenator models predict similar trends with respect to the effect of the emulsion droplet diameter and the dispersed phase volume fraction. Figs. 8 and 9 show the effect of emulsion droplet size on the $\mathrm{O}_{2}$ loading rate. Higher rates were observed for the smaller droplet emulsion in both the gas-sparged and the mem-

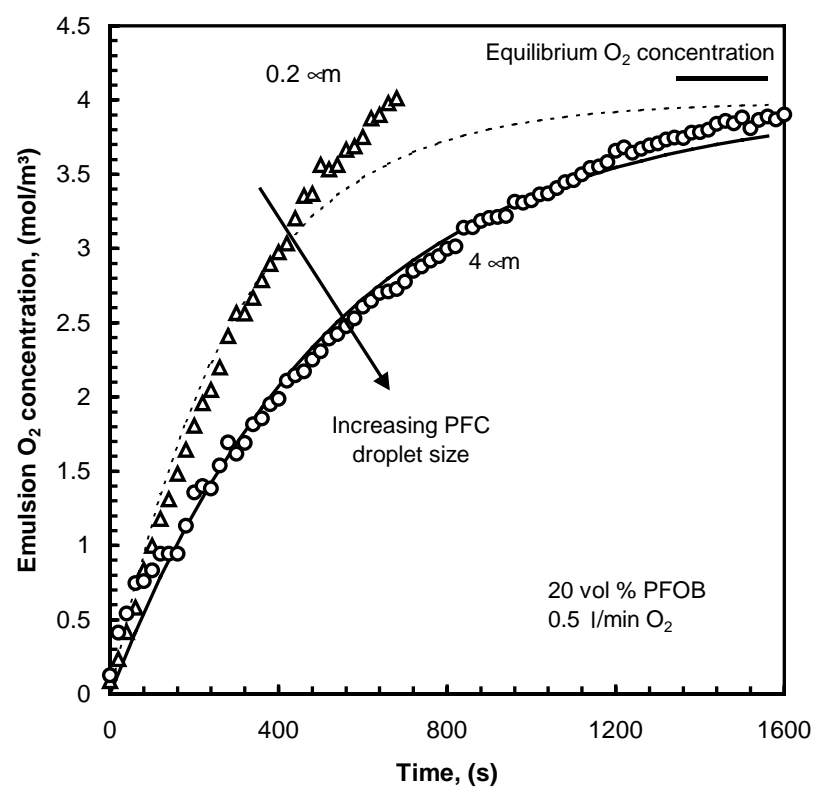

Fig. 8. Gas-sparged oxygenator: effect of emulsion droplet size on oxygen loading rate. Lines show model predictions. Solid horizontal bar indicates equilibrium oxygen concentration.

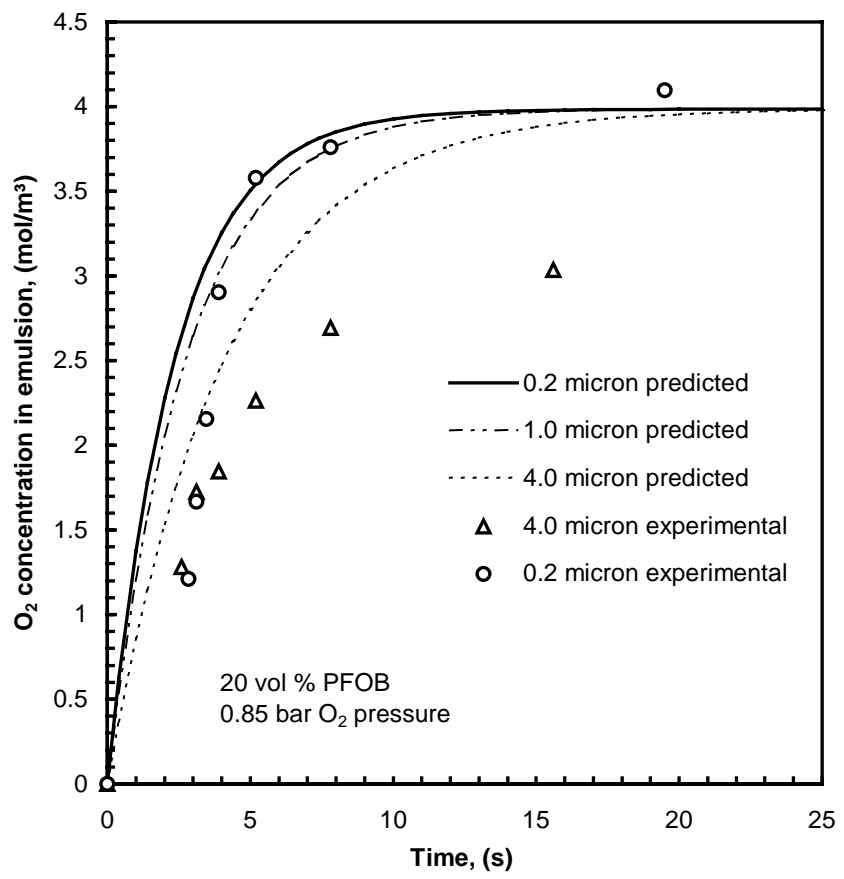

Fig. 9. Membrane oxygenator: effect of dispersed phase droplet diameter on oxygen loading rate.

brane oxygenators. Figs. 10 and 11 show that faster oxygen transfer rates are predicted with increase in PFOB content despite the associated viscosity increase. The data shows much scatter but suggests instead that oxygen saturation is fastest for the 20 vol.\% emulsion and slower at 10 and

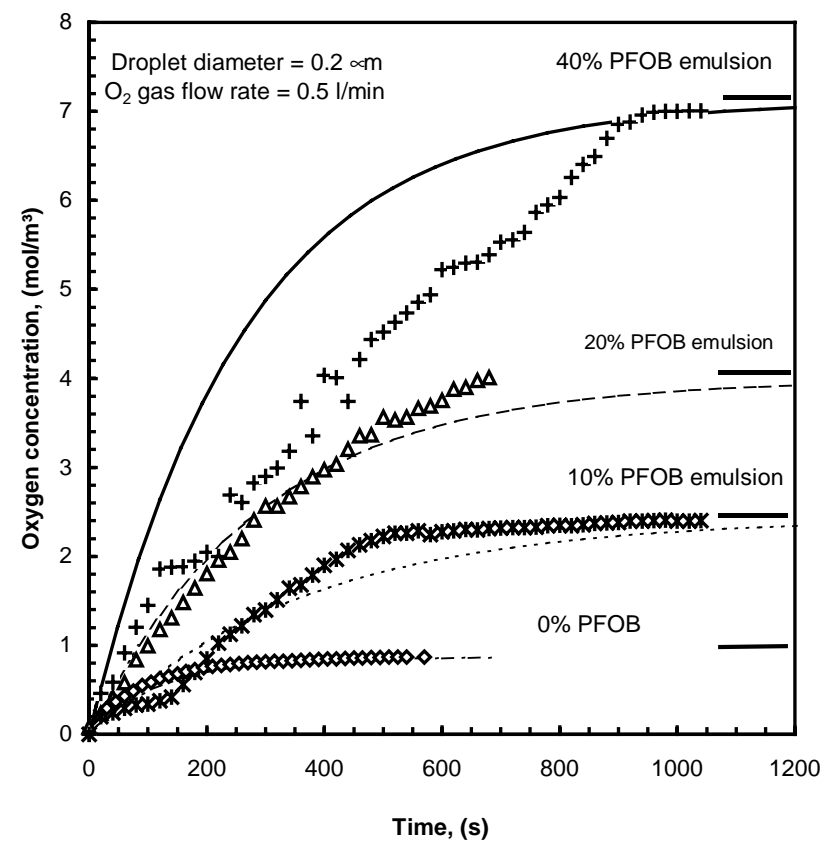

Fig. 10. Gas-sparged oxygenator: effect of dispersed phase volume fraction on oxygen loading rate. Lines show model predictions. Solid horizontal bars indicate equilibrium oxygen concentrations. 


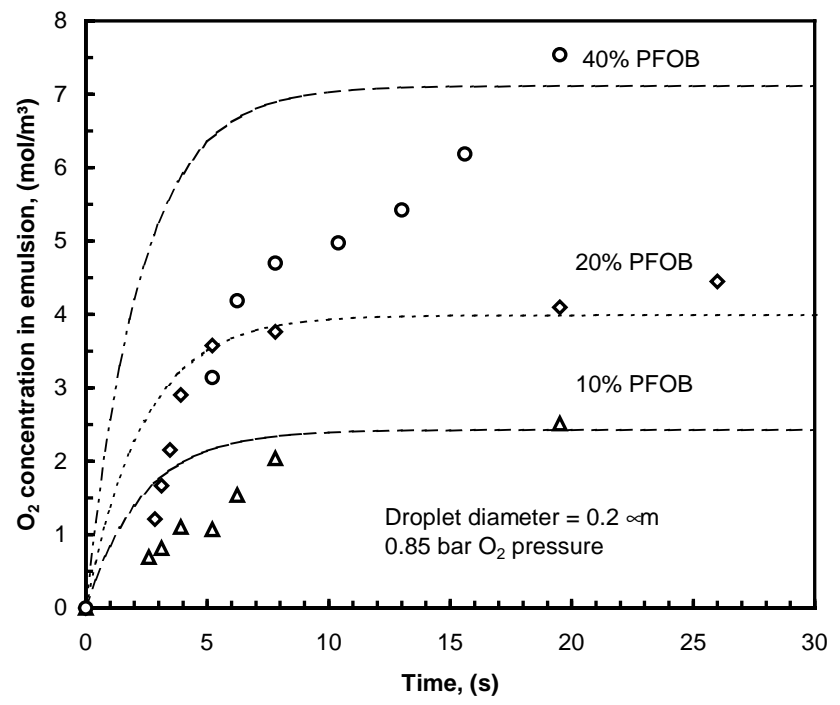

Fig. 11. Membrane oxygenator: effect of dispersed phase volume fraction on oxygen loading rate. Lines show model predictions.

$40 \%$. This suggests optimum mass transfer enhancement at intermediate values of $\phi_{\mathrm{p}}$.

\section{Discussion}

Mehra [17] used Higbie's penetration theory and Danckwerts' penetration theory and developed a comprehensive homogeneous theory for mass transfer in the presence of a dispersed microphase. McMillan and Wang [8] model the transport enhancement in terms of droplet exchange between the boundary layer and the bulk medium. The much simpler modelling approached used here provides reasonable predictions for the effects of droplet size and gas flow rate at 20 vol.\% dispersed phase (see Figs. 6-11). It postulates apparent direct mass transfer between the gas and the dispersed phase. This implies two parallel mass transfer pathways inside the mass transport boundary layer: gas $\leftrightarrow$ medium and gas $\leftrightarrow$ droplet $\leftrightarrow$ medium. The mass transfer coefficient for the second pathway $\left(k_{\mathrm{bp}}\right.$ or $\left.k_{\mathrm{gp}}\right)$ was treated as an adjustable empirical parameter. It was evaluated from experimental data obtained for $20 \mathrm{vol} \%$ PFOB emulsions. Within the uncertainty in the data, the present results suggest that $k_{\mathrm{bp}}$ is independent of the type of reactor (membrane or gas-sparged), the emulsion droplet size $(0.2 \mu \mathrm{m}$ or $4 \mu \mathrm{m})$ or the gas flow rate in the gas-sparged reactor $\left(0.5-11 \mathrm{~min}^{-1}\right)$. However, the results for the 10 and 40 vol.\% PFOB emulsions in the membrane oxygenator are more consistent with lower $k_{\mathrm{bp}}$ values. Interestingly, Elibol and Mavituna [40] observed a maximum in the enhancement of oxygen transfer rate to perfluorodecalin at approximately 20 vol.\% dispersed phase. The rapid increase in emulsion viscosity could explain the reduced transport enhancement above $\phi_{\mathrm{p}} \approx 0.2$ : Higher viscosity retards Brownian motion of emulsion droplets. This slows down the collisions at the gas-medium interface and decreases the replacement rate of fluid elements in the boundary layer. Another possible explanation for the membrane separator is provided by the segregation in small-diameter $(<300 \mu \mathrm{m})$ flow paths that leads to lower volume fraction of the dispersed phase near the wall [12].

Small droplets provide for improved oxygen mass transfer owing to the increase in interfacial surface area. Yamamoto et al. [27] did not consider this effect leading them to the conclusion that addition of PFOB is not beneficial for oxygen mass transfer.

Droplet size is determined by the preparation method. With high-pressure homogenization droplet sizes as small as $0.1-0.2 \mu \mathrm{m}$ can be obtained. As the droplets get smaller, the decrease in droplet size with additional homogenization cycles diminishes [28]; and a lower practical limit is reached at approximately 50-100 $\mathrm{nm}$ [14].

Despite its low toxicity, exposure of patients to PFOB remains a risk factor. It must be removed completely from the plasma returning to the patient. In the UP-CSIR BALSS this separation is accomplished with a membrane unit (see Fig. 1). This sets an additional lower size limit for droplets-filtering-out plasma proteins must be avoided. Bearing these factors in mind, it is concluded that the most favourable Sauter mean diameter for PFOB emulsions is in the range of $0.1-0.2 \mu \mathrm{m}$.

Mass transfer rates for $\phi_{\mathrm{p}}=0.2$ and $\phi_{\mathrm{p}}=0.4$ emulsions were comparable at short time periods. This is in agreement with Ju et al. [9] and Elibol and Mavituna [40], who found optimum enhancement factors $\left(k_{\mathrm{e}} a_{\mathrm{e}} / k_{\mathrm{m}} a_{\mathrm{m}}\right)$ in the range of $\phi_{\mathrm{p}}=0.15-0.2$, with a slight decrease in enhancement factor with further increase in $\phi_{\mathrm{p}}$. However, owing to the higher oxygen carrying capacity of the $\phi_{\mathrm{p}}=0.4$ emulsion, it will maintain higher oxygen concentrations (and thus concentration gradients) for longer periods. Thus, higher dispersed phase volume fractions lead to better overall oxygen mass transfer to cell cultures.

The droplet size distribution determines the maximum volume fraction dispersed phase. It is usually in the range $\phi_{\mathrm{p}}=0.6-0.7$ [27]. However, biological applications mandate emulsions with reasonably low viscosities. Fig. 5 shows that the emulsion viscosity increases by an order of magnitude at $f_{\mathrm{p}} \approx 0.4$ and by two orders magnitude at $\phi_{\mathrm{p}} \approx 0.5$. Over this range the consistency of the emulsion changes from creamy to waxy. This implies that the optimum dispersed phase volume fraction for PFOB emulsions lies in the range $0.4<\phi_{\mathrm{p}}<0.5$. At $\phi_{\mathrm{p}}=0.4$, the emulsion oxygen carrying capacity is 8.3 times higher than that of water/medium alone.

\section{Conclusions}

Mass transfer models for the oxygenation of PFOB emulsions in both gas-sparged and membrane units were developed. Their predictive utility was proven experimentally. 
Consequently, they provide a rational tool for the sizing of oxygenator units in the UP-CSIR BALSS. It was determined that hollow fibre membrane oxygenators provide much better $\mathrm{O}_{2}$ loading efficiency than gas-sparged units. Optimal emulsion properties, based on theoretical and experimental findings, were established. They suggest the following guidelines when preparing PFC emulsions for biological applications: choose a PFC with the highest possible oxygen carrying capacity; maximise the PFC volume fraction to the limit where the emulsion flow properties are still acceptable; and, use the smallest emulsion droplets possible that still allows facile removal with membrane separators.

\section{Acknowledgements}

Financial support from the CSIR and the University of Pretoria is gratefully acknowledged. Thanks are also due to Bauke Vermaas for experimental work on the oxygenators, to Jack Ledwaba for emulsion preparation and Wim Mandersloot for editorial assistance.

\section{Appendix A. Variables and parameters for the gas-sparged oxygenator model}

$\begin{array}{ll}\text { Variable/equation } & \text { Source }\end{array}$

Equilibrium concentration of gas in liquid phase:

$C_{\mathrm{g}}($ liq $)=\frac{P_{\mathrm{g}}}{H_{\mathrm{g}-1}}$

(Henry's law is valid for weakly soluble gases, e.g. $\mathrm{O}_{2}$ [18]. $\mathrm{O}_{2}$ dissolution in both medium and PFOB follows Henry's law [31].)

Henry's constant for oxygen in medium/plasma at $37^{\circ} \mathrm{C}$ :

$H_{\mathrm{O}_{2}-\mathrm{m}}=0.988 \mathrm{bar} \mathrm{m}^{3} / \mathrm{mol}$

Henry's constant for oxygen in $\mathrm{PFOB}$ at $37^{\circ} \mathrm{C}$ :

$H_{\mathrm{O}_{2}-\mathrm{p}}=0.0516 \mathrm{bar} \mathrm{m}^{3} / \mathrm{mol}$

Oxygen distribution coefficient between medium and PFOB:

$K=\frac{C_{\mathrm{p} \text {,equil }}\left(C_{\mathrm{m}, \text { equil }} M_{\mathrm{O}_{2}}+\rho_{\mathrm{m}}\right)}{C_{\mathrm{m}, \text { equil }}\left(C_{\mathrm{p}, \text { equil }} M_{\mathrm{O}_{2}}+\rho_{\mathrm{p}}\right)}$

But $r \gg C M$. Therefore:

$K \approx \frac{H_{\mathrm{O}_{2}-\mathrm{m}} \rho_{\mathrm{m}}}{H_{\mathrm{O}_{2}-\mathrm{p}} \rho_{\mathrm{p}}}$

Specific surface areas of gas bubbles:

Derived [15]

$a_{\mathrm{g}}=\frac{6 \phi_{\mathrm{g}}}{\mathrm{d}_{\mathrm{sg}}}$

Specific surface area of PFOB droplets:

Derived [15]

$a_{\mathrm{p}}=\frac{6 \phi_{\mathrm{p}}\left(1-\phi_{\mathrm{g}}\right)}{\mathrm{d}_{\mathrm{sp}}}$

Interfacial specific surface area between PFOB and gas bubbles:

Estimate [33]

$a_{\mathrm{gp}}=\frac{\phi_{\mathrm{p}}}{0.64} a_{\mathrm{g}}$

(maximum random packing of monodisperse sphere is ca. 0.64)

Interfacial specific surface area between gas and medium:

$a_{\mathrm{gm}}=a_{\mathrm{g}}-a_{\mathrm{gp}}$

From definitions

Interfacial specific surface area between medium and PFOB:

From definitions

$a_{\mathrm{mp}}=a_{\mathrm{p}}-a_{\mathrm{gp}}$

Concentration of oxygen at phase boundaries with gas:

Henry's law

$C_{\mathrm{m}, \mathrm{g}}=\frac{P_{\mathrm{O}_{2}}}{H_{\mathrm{O}_{2}-\mathrm{m}}} ; \quad C_{\mathrm{p}, \mathrm{g}}=\frac{P_{\mathrm{O}_{2}}}{H_{\mathrm{O}_{2}-\mathrm{p}}}$ 
Appendix A (Continued)

Variable/equation

Source

Concentration of dissolved oxygen in medium in equilibrium with PFOB (derived from

Henry's law):

$C_{\mathrm{m}, \text { equil }}=\left(\frac{\rho_{\mathrm{m}}}{\rho_{\mathrm{p}}}\right) \frac{C_{\mathrm{p}}}{K}=\frac{H_{\mathrm{O}_{2}-\mathrm{p}}}{H_{\mathrm{O}_{2}-\mathrm{m}}} C_{\mathrm{p}}$

Mass transfer coefficients: gas to medium and medium to PFOB:

$k_{\mathrm{gm}}=k_{\mathrm{mp}}=\frac{0.13 D_{\mathrm{om}}}{d_{\mathrm{imp}}}\left(\frac{d_{\mathrm{imp}}^{4} \rho_{\mathrm{e}}^{2} P_{\mathrm{ag}}}{\eta_{\mathrm{e}}^{3} V}\right)^{0.25} S c^{0.33}$

Density of emulsion at $37^{\circ} \mathrm{C}$ :

Determined experimentally

$\rho_{\mathrm{e}}=1025+843.7 \phi_{\mathrm{p}}$

Binary diffusion coefficient of oxygen in water at $37^{\circ} \mathrm{C}$ :

$D_{\text {om }}=2.6 \times 10^{-9} \mathrm{~m}^{2} / \mathrm{s}$

Binary diffusion coefficient of oxygen in PFOB:

$D_{\text {op }}=1.86 \times 10^{-18} \frac{M_{\mathrm{p}}^{0.5} T}{\eta_{\mathrm{p}} V_{\mathrm{ob}}^{0.6}}$

Wilke-Chang method: unassociated solvents and low solute concentrations

Gas hold-up in agitated vessel:

$\phi_{\mathrm{g}}=0.105\left(\frac{Q_{\mathrm{g}}}{N_{\mathrm{imp}} \mathrm{d}_{\mathrm{imp}}^{3}}\right)\left(\frac{N_{\mathrm{imp}}^{2} \mathrm{~d}_{\mathrm{imp}}}{g}\right)^{0.5}\left(\frac{N_{\mathrm{imp}} \mathrm{d}_{\mathrm{imp}} \rho_{\mathrm{e}}}{\eta_{\mathrm{e}}}\right)^{0.1}$

Agitator power input: $P_{\mathrm{ag}}=N_{\mathrm{p}} \rho_{\mathrm{e}} N_{\mathrm{imp}}^{3} d_{\mathrm{imp}}^{5}$

Impeller power number (valid for $R e<100000$ ):

$N_{\mathrm{p}}=0.39+8.51 \frac{\ln R e_{\mathrm{imp}}}{R e_{\mathrm{imp}}}+\frac{55.07}{R e_{\mathrm{imp}}^{0.5}}+20.65 \mathrm{e}^{-R e_{\mathrm{imp}}}$

Impeller Reynolds number:

$R e_{\text {imp }}=\frac{\mathrm{d}_{\mathrm{imp}}^{2} N_{\mathrm{imp}} \rho_{\mathrm{e}}}{\eta_{\mathrm{e}}}$

Gas bubble Sauter mean diameter:

$R e_{\mathrm{g}}=\frac{\mathrm{d}_{\mathrm{or}} u_{\mathrm{or}} \rho_{\mathrm{g}}}{\eta_{\mathrm{g}}}$

$d_{\mathrm{sg}}=\left[\frac{\sigma_{\mathrm{s}} \mathrm{d}_{\mathrm{or}}^{2}}{1000\left(\rho_{\mathrm{e}}-\rho_{\mathrm{g}}\right) g}\right]^{0.25}\left(\mathrm{e} R e_{\mathrm{g}}+f\right)$

\section{Appendix B. Additional variables and parameters for the membrane oxygenator model}

Variable/equation

Source

Total internal volume of membrane oxygenator:

By definition

$V_{\mathrm{t}}=V_{\mathrm{gb}}+V_{\mathrm{m}}+V_{\mathrm{p}}+V_{\mathrm{b}}$

Volume ratio (total volume/volume of medium):

Derived [15]

$\frac{V_{\mathrm{t}}}{V_{\mathrm{m}}}=\frac{V_{\mathrm{t}}}{\left(1-\phi_{\mathrm{p}}\right) V_{\mathrm{e}}}=\frac{1}{\left(1-\phi_{\mathrm{p}}\right)\left[1-(\pi / 4) N_{\mathrm{b}} L_{\mathrm{b}}\left(\mathrm{d}_{\mathrm{b}}+2 h_{\mathrm{b}}\right)^{2}\right]}$ 
Volume ratio (total/ PFOB droplet volume):

$\frac{V_{\mathrm{t}}}{V_{\mathrm{p}}}=\frac{V_{\mathrm{t}}}{\phi_{\mathrm{p}} V_{\mathrm{e}}}=\frac{1}{\phi_{\mathrm{p}}\left[1-(\pi / 4) N_{\mathrm{b}} L_{\mathrm{b}}\left(\mathrm{d}_{\mathrm{b}}+2 h_{\mathrm{b}}\right)^{2}\right]}$

Specific surface areas:

$a_{\mathrm{gb}}=\pi N_{\mathrm{b}} L_{\mathrm{b}} d_{\mathrm{b}} \quad a_{\mathrm{b}}=\pi N_{\mathrm{b}} L_{\mathrm{b}}\left(d_{\mathrm{b}}+2 h_{\mathrm{b}}\right)$

$a_{\mathrm{bp}}=\frac{\phi_{\mathrm{p}}}{0.64} a_{\mathrm{b}} \quad a_{\mathrm{bm}}=a_{\mathrm{b}}-a_{\mathrm{bp}}$

$a_{\mathrm{mp}}=a_{\mathrm{p}}-a_{\mathrm{bp}} \quad a_{\mathrm{p}}=\frac{6}{d_{\mathrm{sp}}} \phi_{\mathrm{p}}\left[1-\frac{\pi}{4} N_{\mathrm{b}} L_{\mathrm{b}}\left(d_{\mathrm{b}}+2 h_{\mathrm{b}}\right)^{2}\right]$

Hydraulic radius/diameter:

$d_{\mathrm{h}}=2 R_{\mathrm{h}}=\left(\frac{\mathrm{d}_{\mathrm{i}}^{2}-N_{\mathrm{b}} V_{\mathrm{t}}\left(\mathrm{d}_{\mathrm{b}}+2 h_{\mathrm{b}}\right)^{2}}{\mathrm{~d}_{\mathrm{i}}+N_{\mathrm{b}} V_{\mathrm{t}}\left(\mathrm{d}_{\mathrm{b}}+2 h_{\mathrm{b}}\right)}\right)$

Mass transfer coefficient from membrane into medium for laminar flow:

$S h=\left(S h_{1}^{3}+S h_{2}^{3}+S h_{3}^{3}\right)^{1 / 3}$

With $S h_{1}=3.66+1.2\left(1-\varepsilon_{\mathrm{c}}\right)^{-0.4}$

$S h_{2}=1.615\left(1+0.14\left(1-\varepsilon_{\mathrm{c}}\right)^{-0.25}\right)\left(\frac{\operatorname{Re} S c d_{\mathrm{h}}}{L_{\mathrm{b}}}\right)^{1 / 3}$

$S h_{3}=\left(\frac{2}{1+22 S c}\right)^{1 / 6}\left(\frac{\operatorname{Re} S c d_{\mathrm{h}}}{L_{\mathrm{b}}}\right)^{1 / 2}$

$S h=\frac{k_{\mathrm{bm}} d_{\mathrm{h}}}{D_{\mathrm{o}, \mathrm{m}}} ; \quad S h=\frac{k_{\mathrm{bm}} d_{\mathrm{h}}}{D_{\mathrm{o}, \mathrm{m}}}, \quad R e_{L} \leq 10000 ; \quad S c=\frac{\eta_{\mathrm{e}}}{\rho_{\mathrm{e}} D_{\mathrm{o}, \mathrm{m}}}$

Mass transfer coefficient from medium to PFOB (mass transfer to and from spheres):

$S h=2+\left(0.4 R e^{0.5}+0.06 R e^{0.67}\right) S c^{0.4}\left(\frac{\eta_{\mathrm{e}}}{\eta_{\mathrm{w}}}\right)^{0.25}$

where $S h=\frac{k_{\mathrm{mp}} d_{\mathrm{sp}}}{D_{\mathrm{o}, \mathrm{p}}} ; \quad R e_{D}=\frac{\rho_{\mathrm{e}} u_{\mathrm{e}} \mathrm{d}_{\mathrm{sp}}}{\eta_{\mathrm{e}}}, 3.5 \leq R e_{L} \leq 76000 \quad S c=\frac{\eta_{\mathrm{e}}}{\rho_{\mathrm{e}} \mathrm{D}_{\mathrm{o}, \mathrm{p}}}, 0.71 \leq S c \leq 380$

Emulsion volume average velocity through oxygenator:

Derived [15]

$u_{\mathrm{e}}=\frac{L_{\mathrm{b}}}{\tau_{\mathrm{e}}}$

\section{References}

[1] V. Dixit, G. Gitnick, Artificial liver support: state of the art, Scand. J. Gastroenterol. 31 (Suppl. 220) (1996) 101-114.

[2] A.J. Van Wyk, R.P. Bond, F.S. Moolman, S.W. van der Merwe, Bio-reactor Device, PCT Patent WO02/22775 (2002).

[3] C. Legallais, B. David, E. Doré, Bioartificial livers (BAL): current technological aspects and future developments, J. Membr. Sci. 181 (2001) 81-95.

[4] J.W. Allen, T. Hassanein, S.N. Bhatia, Advances in bioartificial liver devices, Hepatology 34 (3) (2001) 447-455.

[5] K.C. Lowe, P. Anthony, J. Wardrop, M.R. Davey, J.B. Power, Perfluorochemicals and cell biotechnology, Artif. Cells Blood Subst. Immob. Biotechnol. 25 (3) (1997) 261-274.
[6] M.G. Scott, D.F. Kucik, L.T. Goodnough, T.G. Monk, Blood substitutes: evolution and future applications, Clin. Chem. 43 (1997) 1724-1731.

[7] M. Elibol, F. Mavituna, Effect of perfluorodecalin as an oxygen carrier on actinorhodin production by Streptomyces coelicolor, Appl. Microbiol. Biotechnol. 43 (1995) 206-210.

[8] J.D. McMillan, D.I.C. Wang, Enhanced oxygen transfer using oilin-water dispersions, Ann. N.Y. Acad. Sci. 506 (1987) 569-582.

[9] L.K. Ju, J.F. Lee, W.B. Armiger, Enhancing oxygen transfer in bioreactors by perfluorocarbon emulsions, Biotechnol. Prog. 7 (1991) 323-329.

[10] C.T. Wabel, Influence of Lecithin on Structure and Stability of Parenteral Fat emulsions, Ph.D. dissertation, University of ErlangenNürnberg, Germany, 1998.

[11] N.S. Faithfull, R.G. Weers, Perfluorocarbon compounds, Vox Sanguinis 74 (2) (1998) 243-248. 
[12] S. Patel, A. Mehra, Modeling of oxygen transport in bloodperfluorocarbon emulsion mixtures part i: oxygen uptake in tubular vessels, ASAIO 44 (1998) 144-156.

[13] K.C. Lowe, M.R. Davey, J.B. Power, Perfluorochemicals: their applications and benefits to cell culture, Tibtech 16 (1998) 272-277.

[14] M.P. Krafft, J.G. Riess, R.G. Weers, The design and engineering of oxygen-delivering fluorocarbon emulsions, in: S. Benita, Submicron Emulsions in Drug Targeting and Delivery, Harwood Academic Publishers, Amsterdam, 1998.

[15] F.S. Moolman, Oxygen carriers in a novel bio-artificial liver support system, Ph.D. dissertation, University of Pretoria, Pretoria, South Africa, 2004.

[16] L.K. Ju, J.F. Lee, W.B. Armiger, Effect of the interfacial surfactant layer on oxygen transfer through the oil/water phase boundary in perfluorocarbon emulsions, Biotechnol. Bioeng. 37 (1991) 505.

[17] A. Mehra, Intensification of multiphase reactions through the use of a microphase-I, Theoretical Chem. Eng. Sci. 43 (1988) 899-912.

[18] F.P. Incropera, D.P. De Witt, Fundamentals of Heat and Mass Transfer, third ed., Wiley, Singapore, 1990.

[19] H.-J. Lehmler, P.M. Bummer, M. Jay, Liquid ventilation-a new way to deliver drugs to diseased lungs? Chemtech 29 (10) (1999) 7-12.

[20] A.R. Goerke, J. Leung, S.R. Wickramasinghe, Mass and momentum transfer in blood oxygenators, Chem. Eng. Sci. 57 (2002) 2035-2046.

[21] D.G. Zill, M.R. Cullen, Advanced Engineering Mathematics, PWSKENT Publishing Company, USA, 1992.

[22] A.H.P. Skelland, Non-Newtonian Flow and Heat Transfer, Wiley, USA, 1967.

[23] E.L. Cussler, Personal communication, Re: Mass transfer in nonNewtonian systems, 15/03/2002, cuss1001@tc.umn.edu.

[24] W.G.B. Mandersloot, K. Scott, Rheology of particle suspensions, S.A. J. Chem. Eng. 2 (2) (1990) 53-69.

[25] W.B. Dandliker, W.K.R. Watson, T.C. Drees, Artificial blood containing perfluoro chemicals in presence of emulsifying agents, US Patent 5,434,191, granted 18/07/1995.

[26] L.L. Schramm, Emulsions, Fundamentals and Application in the Petroleum Industry, Advances in Chemistry Series 231, American Chemical Society, USA 1992.
[27] S. Yamamoto, N. Shiragami, H. Unno, Analysis of oxygen transfer enhancement by oxygen carrier in the autotrophic cultivation of Alcaligenes eutrophus under low oxygen partial pressure, J. Chem. Eng. Jpn. 27 (4) (1994) 449-454.

[28] J.M. Asua, Miniemulsion polymerization, Progr. Polym. Sci. 27 (2002) 1283-1346.

[29] E.L. Cussler, Diffusion: Mass Transfer in Fluid Systems, Cambridge University Press, USA, 1984.

[30] F. Lipnizki, R.W. Field, Mass transfer performance for hollow fibre modules with shell-side axial feed flow: using an engineering approach to develop a framework, J. Membr. Sci. 193 (2001) 195208.

[31] T.F. Zuck, J.G. Riess, Current status of injectable oxygen carriers, Crit. Rev. Clin. Lab. Sci. 31 (4) (1994) 295-324.

[32] K.C. Lowe, Perfluorochemicals: blood substitutes and beyond, Adv Mater. 3 (2) (1991) 87-93.

[33] H.M. Jaeger, S.R. Nagel, Physics of granular states, Science 255 (1992) 1524.

[34] A.L. Hines, R.N. Maddox, Mass Transfer: Fundamentals and Applications, Prentice-Hall, USA, 1985.

[35] R.H. Perry, D. Green, Perry's Chemical Engineers' Handbook, sixth ed., McGraw-Hill, USA, 1984.

[36] R.H. Perry, D.W. Green, J.O. Maloney, Perry's Chemical Engineers' Handbook, seventh ed., McGraw-Hill Publishing Company, USA, 1997.

[37] D.W.F. Brilman, Mass transfer and chemical reaction in gas-liquid-liquid systems, PhD Thesis, University of Twente, 1998.

[38] D.W.F. Brilman, M.J.V. Goldschmidt, G.F. Versteeg, W.P.M. van Swaaij, Heterogeneuos mass transfer models for gas absorption in multiphase systems, Chem. Eng. Sci. 55 (2000) 2793-2812.

[39] A.A.C.M. Beenackers, W.P.M. van Swaaij, Mass transfer in gas-liquid slurry reactors, Chem. Eng. Sci. 48 (1993) 3109-3139.

[40] M. Elibol, F. Mavituna, Characteristics of antibiotic production in a multiphase system, Proc. Biochem. 32 (1997) 417-422. 